\title{
Spectroscopy and micro-luminescence mapping of Xe-implanted defects in diamond
}

\author{
Y. Deshko and A.A. Gorokhovsky \\ The College of Staten Island and The Graduate Center of CUNY, 2800 Victory Blvd., Staten Island, NY 10314, USA \\ E-mail: Anshel.Gorokhovsky@csi.cuny.edu \\ Received January 28, 2010
}

\begin{abstract}
Ion implantation in diamond creates optically active defects which have emission lines in broad spectral regions, and may be used in advanced photonics and optical communication applications. A brief review of the photoluminescence properties of $\mathrm{Xe}^{+}$ion implanted diamond is presented. The Xe-related center is of particular interest as this center is one of a few centers $(\mathrm{Ni}, \mathrm{Si}, \mathrm{Cr})$ in diamond having sharp emission lines in the infrared spectral region, specifically at 813 and $794 \mathrm{~nm}$. The paper discusses an approach to determine an important and difficult to measure conversion efficiency of implanted ions into emitting optical centers. The method uses a micro-luminescence confocal mapping and statistical analysis based on a compound Poisson distribution, accounting for both the implanted centers and the optically excited centers statistics. Results of numerical simulations and experimental measurements are presented.
\end{abstract}

PACS: 78.55.-m Photoluminescence, properties and matherials;

73.20.Hb Impurity and defect levels; energy states of adsorbed species.

Keywords: photoluminescence, infrared spectroscopy, implanted ions.

\section{Introduction}

Diamond is an extremely hard, highly thermally conducting, and chemically inert material. It has the optical transparency in the visible and near-infrared regions of the spectrum and is widely used for optical windows in critical applications. The optical properties of diamond are significantly influenced by optically active defect centers. There are several hundreds of intrinsic as well as introduced optical luminescence centers in diamond which have very different spectral features like positions, band-widths, intensities, quantum efficiencies, temperature behavior, and etc., and many of the features of these centers have been described in past reviews [1,2]. In recent years a lot of attention has been paid to a few optical defect centers in diamond with high quantum efficiency and which are sufficiently photo-stable under laser excitation to successfully serve as single photon emitters at room temperatures. Single photon generation was recently demonstrated on NV (nitrogen-vacancy) centers [3], Ni-related centers [4], Sirelated centers [5], and Cr-related centers [6].

The technique of ion implantation and postimplantation annealing allows one to introduce into diamond different defect centers having emission lines in broad spectral regions, including near-infrared. Another advantage of ion implantation is the possibility to control the dose and the energy of the ions, which gives a robust tool for device fabrication. It was shown that the NV center pattern can be written on a diamond surface with high spatial accuracy [7], and even a single NV center may be generated by focused nitrogen implantation $[8,9]$. On the other hand, ion implantation creates a number of radiation defects, mainly $s p^{2}$ bonds that modify the properties of diamond and influence optical emission from the centers. Spectroscopic studies of implanted centers in diamond are important for the better understanding of the defect structure and the interaction of defects with the lattice and with light. Also, they are aimed at the improvement of existing materials and for the search for new potentially useful materials for advanced photonics applications.

In this paper, we briefly review spectroscopic properties of $\mathrm{Xe}^{+}$ion implanted diamonds. As well, we discuss the confocal micro-luminescence mapping of this material with the purpose to estimate the density of emitting centers and the conversion efficiency of implanted atoms into emitting centers. Our interest in the Xe optical center originated from the fact that this center is one of only a few centers having sharp emission spectra in the infrared spectral region. This feature makes this center interesting for applications such as optically driven single photon sources [3-6]. Moreover, a diamond light emitting diode activated 
with Xe centers has been recently reported [10], demonstrating that electrically driven single photon sources could be potentially fabricated.

\section{Luminescence spectra of the Xe center in diamond}

A few papers have been published on the optical properties of the Xe center in diamond [11-14]. A brief summary of the relevant results is presented below. The samples under investigation were natural diamonds (type IIa) and single crystal CVD diamonds. Crystals were irradiated with $500 \mathrm{keV}$ and $180 \mathrm{keV} \mathrm{Xe}{ }^{+}$ions at room temperature within the dose range $10^{10}-5 \cdot 10^{14} \mathrm{ion} / \mathrm{cm}^{2}$. Post-implantation thermal annealing for 1-2 hours was carried out at temperatures up to $T_{A}=1400{ }^{\circ} \mathrm{C}$ under high vacuum conditions.

\subsection{The Xe center photoluminescence}

The low-temperature photoluminescence spectrum of the $\mathrm{Xe}^{+}$implanted diamond (dose $10^{13} \mathrm{ion} / \mathrm{cm}^{2}$ ) after postimplantation thermal treatment at $T_{A}=700{ }^{\circ} \mathrm{C}$ is shown in Fig. 1, $a$ [11]. The lines at $575 \mathrm{~nm}$ (nitrogen related) and at $741 \mathrm{~nm}$ (GR1 center) are clearly seen on the broad background. No Xe-related luminescence line was observed for the annealing temperatures below $800{ }^{\circ} \mathrm{C}$. As the annealing temperature was raised from $800{ }^{\circ} \mathrm{C}$, the zero phonon line (ZPL) at $811.7 \mathrm{~nm}$ was detected, and its intensity grew rapidly with temperature in all samples. The spectrum of the same sample after annealing at $T_{A}=1400{ }^{\circ} \mathrm{C}$ is shown

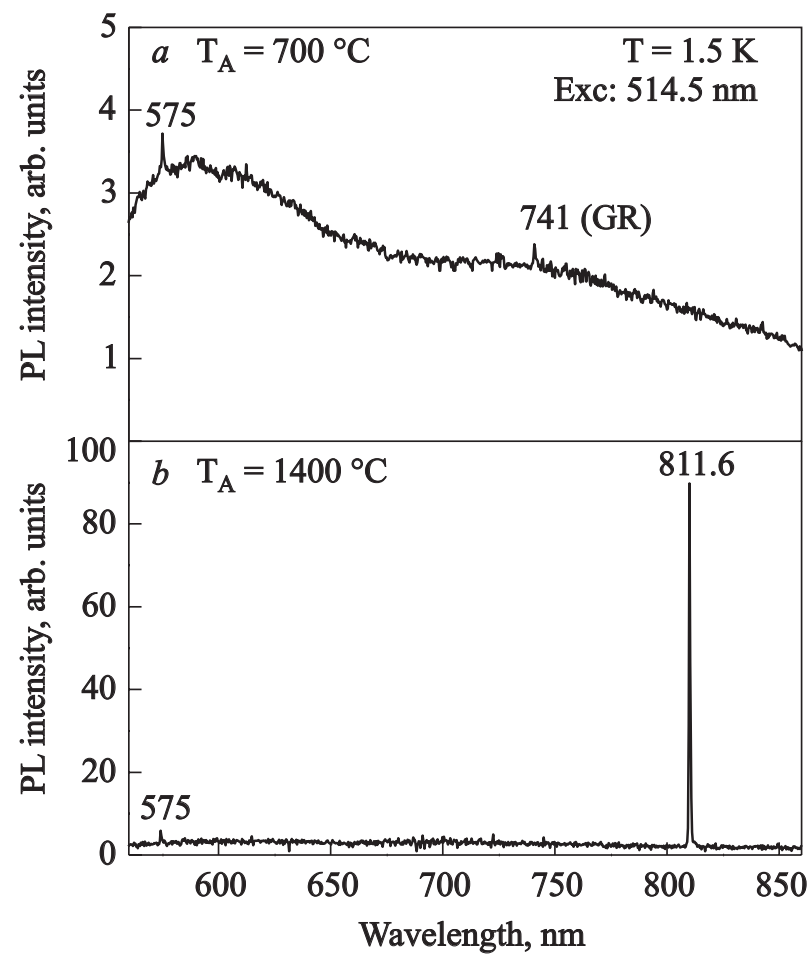

Fig. 1. Photoluminescence spectra measured at $1.5 \mathrm{~K}$ of a diamond implanted with $500 \mathrm{keV}$ Xe ions at a dose of $10^{13} \mathrm{~cm}^{-2}$ and subsequently annealed at: $700{ }^{\circ} \mathrm{C}(a) ; 1400{ }^{\circ} \mathrm{C}(b)$. Excitation wavelength is $514.5 \mathrm{~nm}$. Reprinted from Ref. 11 with permission from Elsevier. in Fig. 1,b. The strong and narrow (width of $5.5 \mathrm{~cm}^{-1}$ ) Xerelated ZPL is seen, and the line at $741 \mathrm{~nm}$ disappeared as the GR1 center was annealed out. Because the $800{ }^{\circ} \mathrm{C}$ is usually considered as the temperature that the isolated vacancies in diamond become mobile, the appearance of the $811.7 \mathrm{~nm}$ line after annealing at $800{ }^{\circ} \mathrm{C}$ suggests that the vacancies are involved in the formation of the Xe centers.

The representative luminescence spectrum at room temperature of the $\mathrm{Xe}^{+}$ion implanted CVD diamond is presented in Fig. 2, $a$ [14]. The spectrum shows the Xe center related features: two ZPLs at $813 \mathrm{~nm}$ and at $794 \mathrm{~nm}$, and a corresponding vibrational sideband. In comparison to the low-temperature spectrum, the ZPL at $811.7 \mathrm{~nm}$ was shifted to $813 \mathrm{~nm}$ and became broader $\left(130 \mathrm{~cm}^{-1}\right)$, and the vibrational sideband became much more visible. The
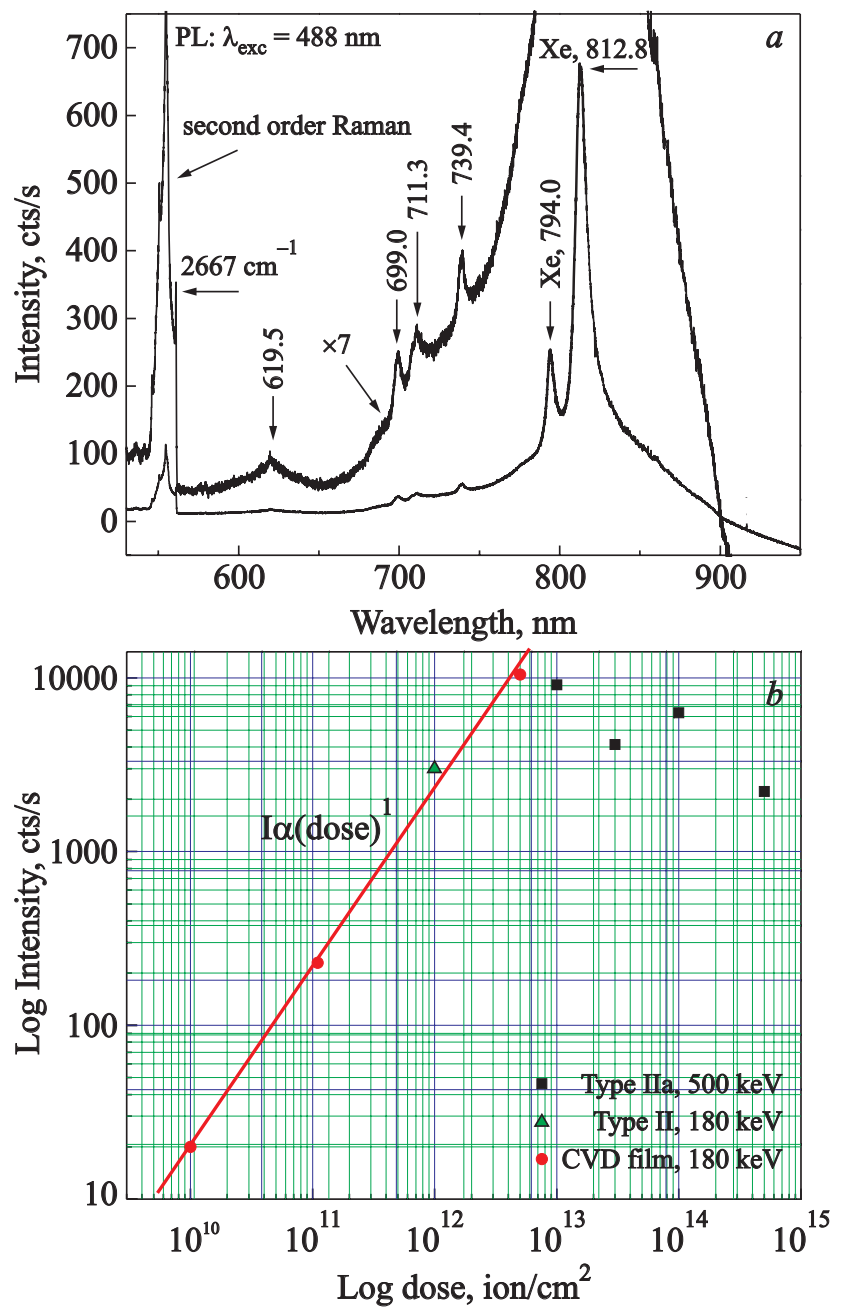

Fig. 2. (a) The representative emission spectrum of the Xe ion implanted single crystal CVD film measured at $300 \mathrm{~K}$. Implantation dose $5 \cdot 10^{12}$ ion $/ \mathrm{cm}^{2}$, laser excitation at $488 \mathrm{~nm}$. (b) The dependence of the intensity of ZPL at $813 \mathrm{~nm}$ on the $\mathrm{Xe}^{+}$ion implantation dose. Data were taken for: circles - CVD diamond film implanted with $180 \mathrm{keV} \mathrm{Xe}{ }^{+}$; triangle - natural diamond (type IIa) implanted with $180 \mathrm{keV} \mathrm{Xe}{ }^{+}$; squares — four natural (type IIa) diamonds irradiated with $500 \mathrm{keV} \mathrm{Xe}{ }^{+}$ions. The solid line represents the best fit by a linear dependence on the dose. Reprinted from Ref. 14 with permission from Elsevier. 
prominent new feature is the growth of the second ZPL at $794 \mathrm{~nm}$ in the anti-Stokes region of the primary ZPL at $813 \mathrm{~nm}$. This line is observed at temperatures above $65 \mathrm{~K}$ and will be discussed in the next section. The DebyeWaller factor, which is a measure of the linear electronphonon coupling, can be determined as a ratio of the integral intensities of ZPL and the total vibronic spectrum (ZPL + sideband) [15]. This factor was estimated as $\exp (-S)=0.9$ at $T=8 \mathrm{~K}[12]$, and 0.3 at $300 \mathrm{~K}$ [13]. This value reflects a low coupling strength as compared to several other defects in diamond $[1,16,17]$. In addition to the Xe center luminescence, the broad background band starting at $700 \mathrm{~nm}$, a few weak lines and the second order Raman complex line at $550 \mathrm{~nm}$ with the sharp peak at $2667 \mathrm{~cm}^{-1}$ is seen in the spectrum as well. The observed structure of the Raman line is related to the critical points of the phonon dispersion curves and to the maxima in the vibrational density of states of diamond [18].

The dependence of the ZPL at $813 \mathrm{~nm}$ intensity on the implantation dose was measured using several crystals with $\mathrm{Xe}^{+}$implantation within the dose range $10^{10}$ $5 \cdot 10^{14}$ ion $/ \mathrm{cm}^{2}$ (see Fig. 2,b) [14]. The dependence obviously consists of two regions. For doses between $10^{10}$ and $5 \cdot 10^{12} \mathrm{ion} / \mathrm{cm}^{2}$, the luminescence intensity grows linearly with the implantation dose. This important result indicates that only a single $\mathrm{Xe}$ atom is incorporated into the optical Xe center. At dose $10^{13} \mathrm{ion} / \mathrm{cm}^{2}$, the intensity saturates and even starts to decrease at higher doses. The observed behavior may be qualitatively explained by quenching of the photoluminescence by the ion radiation-induced defects in our crystals. This nonradiative quenching, apparently, is more significant for the more heavily implanted and therefore more damaged samples. Moreover, the irradiation induced defects play a significant role in the inhomogeneous broadening of the ZPL at $811.7 \mathrm{~nm}$. It was observed at $T=1.5 \mathrm{~K}$ [11] that the ZPL broadened from width of $5.5 \mathrm{~cm}^{-1}$ for dose of $10^{13}$ ion $/ \mathrm{cm}^{2}$ to $15 \mathrm{~cm}^{-1}$ for the $5 \cdot 10^{14}$ ion $/ \mathrm{cm}^{2}$ sample. These results clearly show that this ZPL is broadening inhomogeneously (thermal broadening is small) due to the random internal fields from the radiation induced defects like points defects, dislocations, and agglomerated defects, as such radiation induced defects may withstand thermal annealing.

\subsection{The anti-Stokes luminescence}

As was mentioned above, while at low temperature a single ZPL at $811.7 \mathrm{~nm}$ line was observed, upon heating the sample the second ZPL at $793.1 \mathrm{~nm}$ appeared in the anti-Stokes region of the primary ZPL and continued to grow with increasing temperature (Fig. 3,a) [11,12]. This behavior indicates presence of two excited states with energy splitting equal to the spectroscopic splitting $\Delta E_{\mathrm{sp}}=$ $=E_{793.1}-E_{811.7}=289 \mathrm{~cm}^{-1}$ (see Fig. 4). Using laser excitation in resonance with these optical transitions, it was established that both ZPLs under discussion are resonance
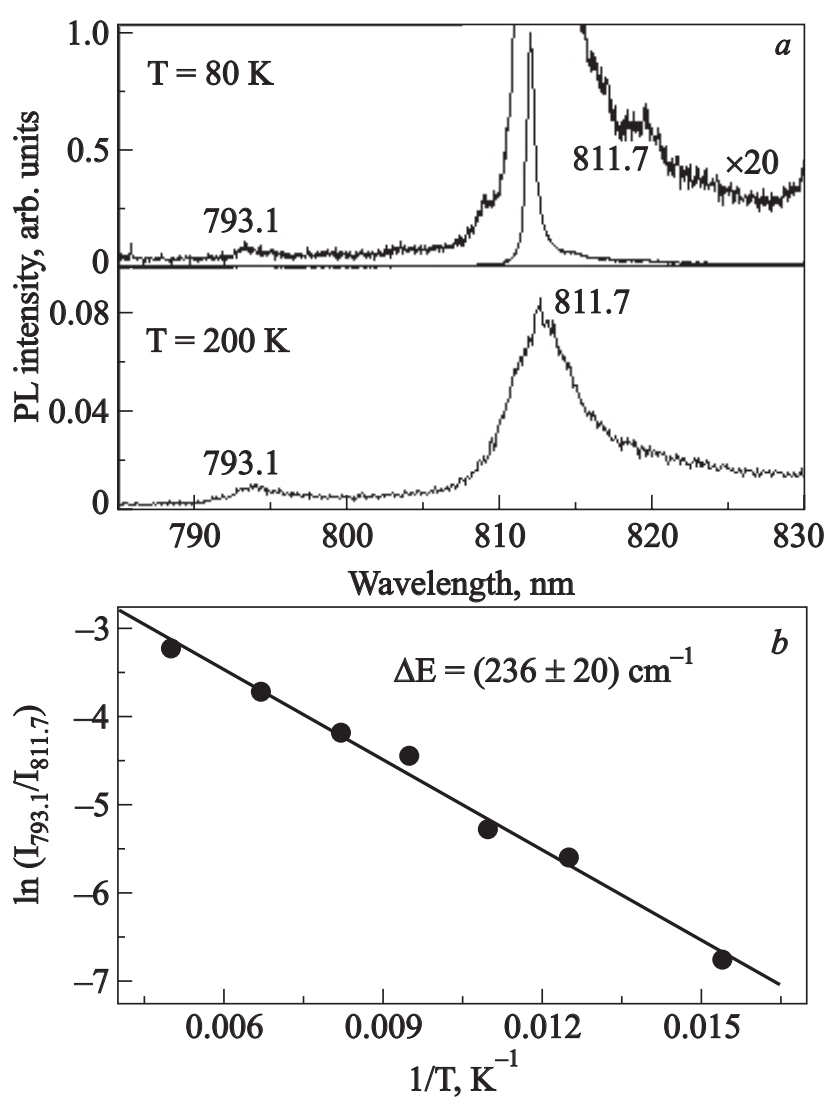

Fig. 3. (a) Photoluminescence spectra of Xe implanted diamond in the region of ZPL at 811.7 and $793.1 \mathrm{~nm}$ measured at 80 and $200 \mathrm{~K}$ with excitation at $514.5 \mathrm{~nm}$. (b) Logarithm of the ratio of the integral intensities of the ZPL at $793.1 \mathrm{~nm}, I_{h}$, and the ZPL at $811.7 \mathrm{~nm}, I_{l}$, plotted against reciprocal temperature. The slope of the line is $\Delta E=(236 \pm 20) \mathrm{cm}^{-1}$. Reprinted from Ref. 12 with permission from Elsevier.

$0-0$ lines [12]. The dependence of the photoluminescence spectra on temperature between 65 and $200 \mathrm{~K}$ is shown in Fig 3,b. We expect the following relation to hold for the intensities $I_{l}$ and $I_{h}$, of the ZPLs, originating from

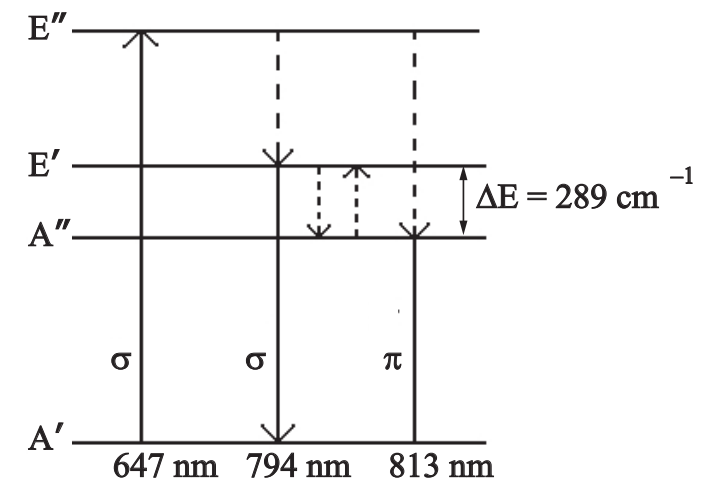

Fig. 4. The Xe center schematic energy level diagram of the ground and excited electronic states. The vertical solid arrows show the laser light absorption and the luminescence transitions, $\pi$ and $\sigma$ notations refer to the type of electrical dipole absorption or emission oscillators. The vertical dotted arrows show phonon transitions. The superscript notations are for labeling purpose only. Reprinted from Ref. 13 with permission from Elsevier. 
the low and high energy levels, respectively: $I_{h} / I_{l}=$ $=\left(g_{h} / g_{l}\right) \exp (-\Delta E / k T)$. Here $\Delta E$ is the energy splitting of the excited state, and $g_{h} / g_{l}$ is the ratio of the respective degeneracies. The logarithmic plot of $I_{h} / I_{l}$ vs $1 / T$ (see Fig. 3,b) agrees well with this scheme. The slope of the line gives a value $\Delta E=236 \mathrm{~cm}^{-1}$ which is apparently less than the spectroscopic splitting $\Delta E_{\mathrm{sp}}=289 \mathrm{~cm}^{-1}$. Several reasons may be responsible for this discrepancy, primary the sample overheating by a laser beam and uncertainty in the determination of the integral intensity of the ZPL, particularly at higher temperatures.

\subsection{The polarized photoluminescence}

We applied the polarized photoluminescence technique $[19,20]$ to establish the site symmetry of the Xe center in diamond. The sample studied was a single crystal CVD film grown along the (001) crystallographic plane and implanted with $\mathrm{Xe}^{+}$at dose of $5 \cdot 10^{12} \mathrm{ion} / \mathrm{cm}^{2}$ [13]. The polarized luminescence spectra were measured as a function of the sample rotation about the crystallographic direction
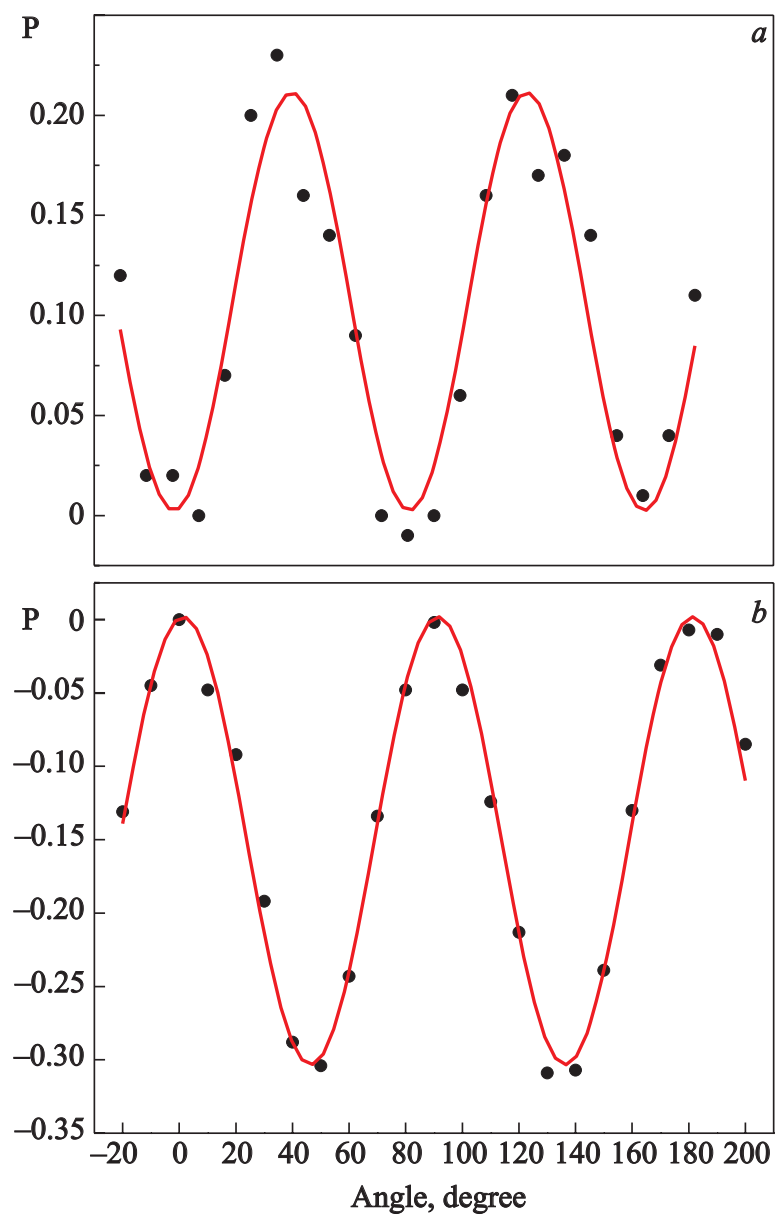

Fig. 5. The degree of polarization (points) for the 794 (a) and 813 (b) nm emission components from the Xe implanted diamond excited by the laser light at $647 \mathrm{~nm}$. Fitting curves are shown. At the angle of rotation, $\theta=0^{\circ}$, the crystallographic direction [010] is parallel to the emitted light analyzer. Reprinted from Ref. 13 with permission from Elsevier.
[001] for the two incident laser light at $647 \mathrm{~nm}$ polarizations — parallel $\left(I_{\|}\right)$and perpendicular $\left(I_{\perp}\right)$ to the emitted light analyzer. The peak intensities of the ZPL at 794 and $813 \mathrm{~nm}$ were determined, and the degree of luminescence polarization was calculated: $P=\left(I_{\|}-I_{\perp}\right) /\left(I_{\|}+I_{\perp}\right)$ [19].

Experimental results for the degree of polarization on angle $P(\theta)$ and the fitting curves are presented in Fig. 5 for both ZPLs. Comparison of the experimental $P(\theta)$ and model calculations for different center orientations and different types of optical transitions shows that the Xe center is oriented along $<111>$ direction, and the luminescence line at $794 \mathrm{~nm}$ is $\sigma-\sigma$ type and line at $813 \mathrm{~nm}$ is $\sigma-\pi$ type. Thus it may be concluded that the Xe center is a trigonal center with $C_{3}$ axis along the $<111>$ direction. For the line at $794 \mathrm{~nm}$, the maximum observed degree of polarization 0.21 is close to the model value of 0.25 for the $\sigma-\sigma$ transitions. For the $813 \mathrm{~nm}$ component, the observed degree of polarization -0.31 is consistent with the transitions $\sigma-\pi$, though it is noticeably less than the model value of -0.5 .

Optical transitions in trigonal centers involve states with irreducible representations $A$ and $E$ [21]. Transitions between $A$ states are $\pi$ type, and between $A$ and $E$ states are $\sigma$ type. The possible energy level scheme is shown in Fig. 4 for the $A$ symmetry ground state. The laser light at $647 \mathrm{~nm}$ is absorbed from the ground state $A^{\prime}$ by a $\sigma$ transition to the upper excited state $E^{\prime \prime}$, the excitation nonradiatively decays from the $E^{\prime \prime}$ level to the lower levels $E^{\prime}$ and $A^{\prime \prime}$, and is followed by radiative transitions $\sigma$ at $794 \mathrm{~nm}$ and $\pi$ transition at $813 \mathrm{~nm}$, respectively. The splitting between the $\mathrm{A}^{\prime \prime}$ and $\mathrm{E}^{\prime}$ levels is equal to the spectroscopic splitting of $\Delta E_{\mathrm{sp}}=289 \mathrm{~cm}^{-1}$, thus the level $E^{\prime}$ is populated by the establishing of thermal equilibrium with the $A^{\prime \prime}$ level.

\subsection{Structure of the Xe center}

Based on discussed results, a single Xe atom and a vacancy (or vacancies) form the Xe center. In addition, this center has a trigonal symmetry. We may propose a few models for the center. The simplest model involves a single substitutional $\mathrm{Xe}$ atom aggregated with the nearest vacancy which forms a $\mathrm{Xe}-\mathrm{V}$ defect of trigonal symmetry, similar to the well known nitrogen related $\mathrm{N}-\mathrm{V}$ center [12]. A model involving a substitutional Xe atom and three adjacent vacancies $\mathrm{Xe}-\mathrm{V}_{3}$, is also feasible. Both these structures have symmetry $C_{3 v}$. Taking into account the large size of the Xe atom, the most likely configuration of the $\mathrm{Xe}$ center is a semi-divacancy site, $\mathrm{V}-\mathrm{Xe}-\mathrm{V}$, oriented along $<111>$ direction. This structure is a stable configuration as was calculated in consideration of the Xe defect in diamond as a potential $n$-type donor [22]. The symmetry of the semi-divacancy structure is $D_{3 d}$ as it has a center of inversion. We expect that the presence of the inversion center may be verified experimentally by Stark-effect measurements; such measurements are in preparation in our laboratory. 


\section{Confocal luminescence mapping and the number of emitting centers}

The probability of the emitting center generation is characterized by an important and difficult to measure conversion efficiency of implanted ions into emitting optical centers. This quantity is particularly important in deterministic single ion doping [8] and quantum devices fabrication by ion implantation [23]. The yield is determined as a ratio of the average volume density of emitting centers to the density of implanted centers. We used the method of confocal micro-luminescence mapping and statistical analyses based on a compound Poisson distribution to determine the conversion efficiency of implanted $\mathrm{Xe}^{+}$ions into Xe optical centers.

\subsection{Flat-top laser beam, case of Poisson distribution}

Physical parameters of the distribution of implanted ions inside diamond can be effectively simulated by SRIM codes [24]. In our case of $180 \mathrm{keV} \mathrm{Xe}^{+}$ions, the implanted atoms form a thin two-dimensional layer located at $68 \mathrm{~nm}$ (ion range) below a diamond surface with thickness of $9.6 \mathrm{~nm}$ (straggling). In the confocal microscopy, a laser beam is focused at the sample with the spot diameter of about $1 \mu \mathrm{m}$, much more than a thickness of the implanted layer. Thus, instead the volume density, one may consider the surface density of the emitting centers. The number of emitting centers may be estimated taking into account the statistical nature of ion implantation and the Poisson law of distribution of the number of implanted centers. In this case, the average number of emitting centers $\bar{N}$ and its fluctuation $\sigma_{N}$ are related: $\sigma_{N}=(\bar{N})^{1 / 2}$. The measured quantity is the luminescence intensity $I$ which is proportional to $N$. By mapping a sufficiently large area, one may determine the average intensity $\bar{I}=I_{1} \bar{N}$ and the fluctuation of intensity $\sigma_{I}=I_{1} \sigma_{N}=I_{1}(\bar{N})^{1 / 2}$, where $I_{1}$ is the signal from a single center. So both $\bar{N}$ and $I_{1}$, may be found from experimental results as $\bar{N}=\left(\bar{I} / \sigma_{I}\right)^{2}$ and $I_{1}=\sigma_{I}^{2} / \bar{I}$. This simple approach is justified only if each optical center has the same $I_{1}$, and the distribution of laser intensity across a focal spot is flat (flat-top beam) (Fig. 6,a).

\subsection{Gaussian beam, case of compound Poisson distribution}

Unfortunately, in real world experiment, these conditions difficult to fulfill, so more complex analysis must be applied. First, due to the anisotropy and the orientational distribution of the optical centers in diamond, experimental conditions must be chosen to make the luminescence intensity of the centers in each orientation equal. For the Xe center in diamond, the polarization of laser beam along [001] and detection analyzer along [010] crystallographic directions assure equal rate of excitation and detection for all centers oriented along all four $<111>$ directions in the cu-

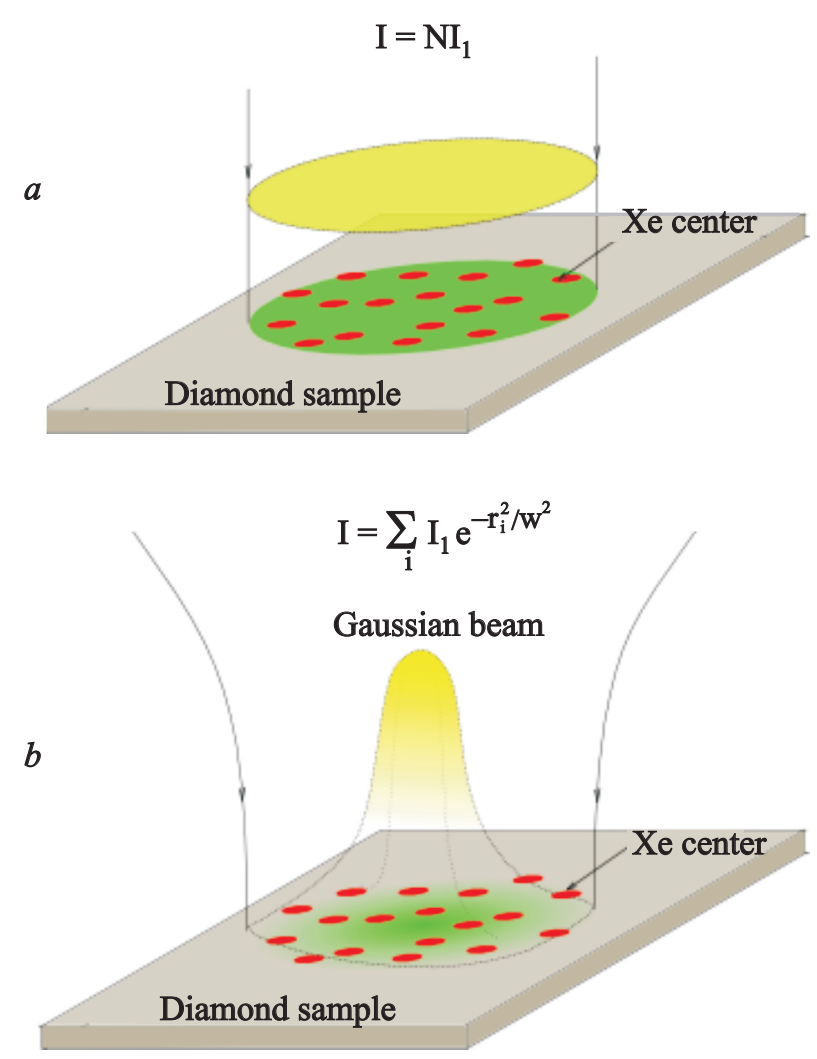

Fig. 6. The intensity distribution in the laser spot are shown for case of (a) Flat-top laser beam that corresponds the Poissonian statistics of the signal, and (b) Gaussian laser beam that corresponds the compound Poissonian statistics of the signal. Radius of the laser $\operatorname{spot} R=2 w$.

bic lattice. Secondly, distribution of the laser intensity inside the focal spot should be accounted for. If this distribution is Gaussian (Fig. 6,b) with the radius of the laser beam waist $w$ in the focal plane, the signal from a single ion located in a random position $r_{i}$ is $I_{1}\left(r_{i}\right)=I_{1} \exp \left[-\left(r_{i} / w\right)^{2}\right]$, where $I_{1}$ is the signal from a single center located in the center of the beam. The total signal from all excited centers is the sum of signals from every center in a random position within the laser spot, $I=\sum I_{1}\left(r_{i}\right)=$ $=I_{1} \sum \exp \left[-\left(r_{i} / w\right)^{2}\right]$. The upper limit in this sum is the total number of centers in the laser spot $N$, which is a random Poisson - distributed number. The total signal $I$ is a random quantity and its properties are described by a compound Poisson distribution [25]. Results of detail analysis will be described elsewhere, but the summary is the following: The relationship between $\bar{N}$ and experimentally measured quantities, $\bar{I}$ and $\sigma_{I}$, for the compound Poisson distribution is: $\bar{N}=\beta\left(\bar{I} / \sigma_{I}\right)^{2}$. Here the parameter $\beta=$ $=\left(k^{2} / 2\right) \operatorname{coth}\left(k^{2} / 2\right)$, and $k=R / w$ is a dimensionless radius of the laser spot which determines the portion of all centers contributing into the total signal. Choice of $k$ is determined 
by the signal/noise ratio in an actual experiment: for higher signal/noise ratio, one may collect a useful luminescence from the larger portion of a laser spot. For the central part of the laser spot with $R=w, k=1$, so $\beta \approx 1$. In this case, the centers inside the spot contribute only $67 \%$ of the total signal and statistics of a signal is close to a simple Poisson. For more realistic case of $R=2 w(k=2)$, when the centers inside the spot contribute about $99 \%$ of the measured signal, $\beta=2.07$.

\subsection{Numerical simulations and experimental results}

The consideration above is illustrated by two numerical simulations for cases of the flat and Gaussian intensity distributions in the laser spot. Results are presented in Fig. 7. The anisotropy of the optical centers, optical saturation and detection noise are not accounted for. The simulated distributions show results of $50 \times 50$ measurements of luminescence intensity from $\bar{N}=1000$ emitting centers randomly distributed inside laser spot with radius $R=2 w$ and having the single center intensity $I_{1}=1$. In the case of a flat top laser intensity, the simulated distribution (Fig. 7,a) is
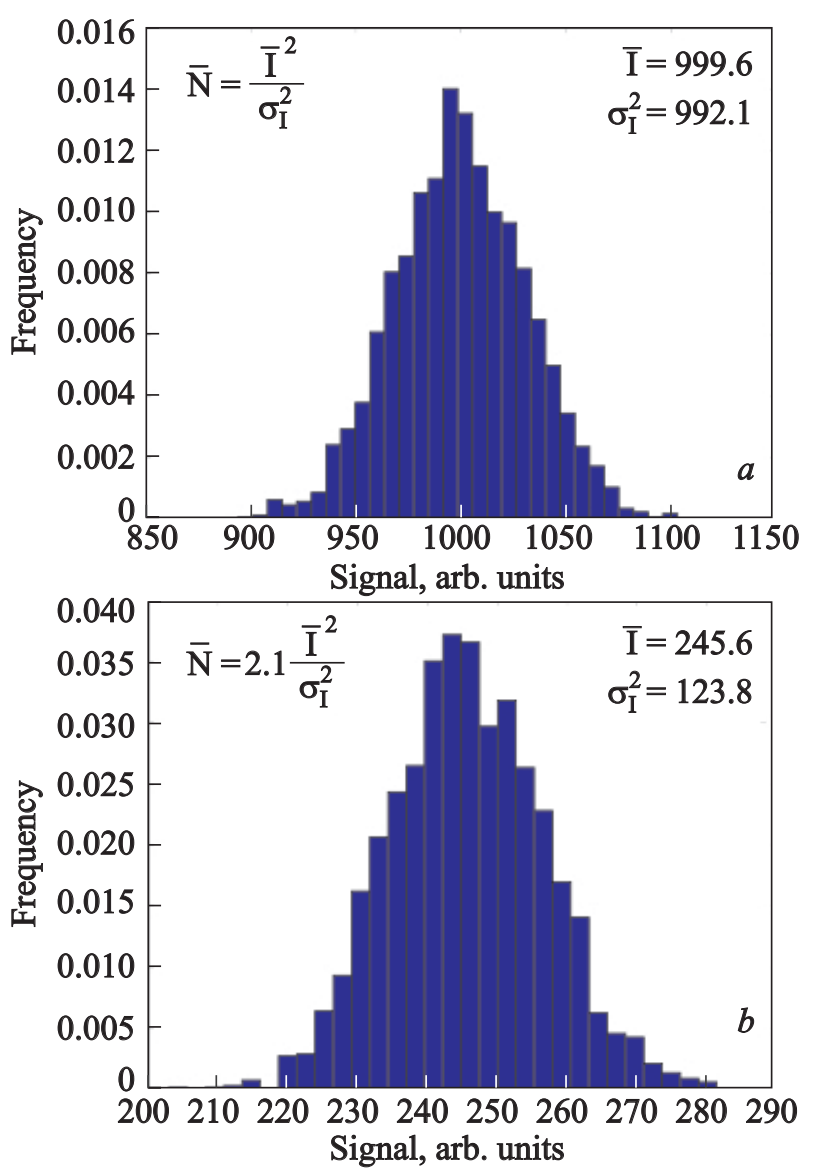

Fig. 7. Histograms of the signal based on the numerically generated map $50 \times 50$ points with the average number of centers inside the laser spot with radius $R=2 w, \bar{N}=1000$, and $I_{1}=1$. It is assumed that the number of centers has a Gaussian distribution with the average $\bar{N}$ and the fluctuation $(\bar{N})^{1 / 2}$. The laser beam profile is a flat-top (constant intensity across the laser spot) for simulation $(a)$ and a Gaussian profile, $\exp \left[-(r / w)^{2}\right]$, for simulation $(b)$. close to a Poisson with $\bar{I}=999.6$ and $\sigma_{I}^{2}=992.1$, so $\bar{N}=$ $=992.1 \approx 1000$, as expected. In the case of the Gaussian laser beam, the simulated distribution gives $\bar{I}=245.6$ and $\sigma_{I}^{2}=123.8$. Using these values and the parameter $\beta=$ $=2.07$, one may calculate the simulated average number of the emitting centers: $\bar{N}=2.07\left(I / \sigma_{I}\right)^{2}=1008.6$, in a good correspondence with the model value of 1000 .

The experimental micro-luminescence mapping was performed for the $\mathrm{Xe}^{+}$ion implanted diamond with the dose of $10^{10}$ ion $/ \mathrm{cm}^{2}$. Spectra were collected in a backscattering geometry using a spectrometer equipped with a confocal microscope and the aberration corrected objective $\times 60$, N.A. $=0.7$, a XYZ scanning stage, a half-wave plate to control polarization of the incident light, an analyzer in the emitted light pass, and a cooled CCD detector. The excitation laser was an $\mathrm{Ar}-\mathrm{Kr}$ laser operating at $514.5 \mathrm{~nm}$, polarization conditions were chosen as discussed in Sec. 3.2. To increase the signal/noise ratio, the sample was placed in the optical cryostat and cooled to temperature $T=80 \mathrm{~K}$. The radius of the laser spot for this arrangement was determined to be $w=0.7 \mu \mathrm{m}$ on the $1 / e$ level.
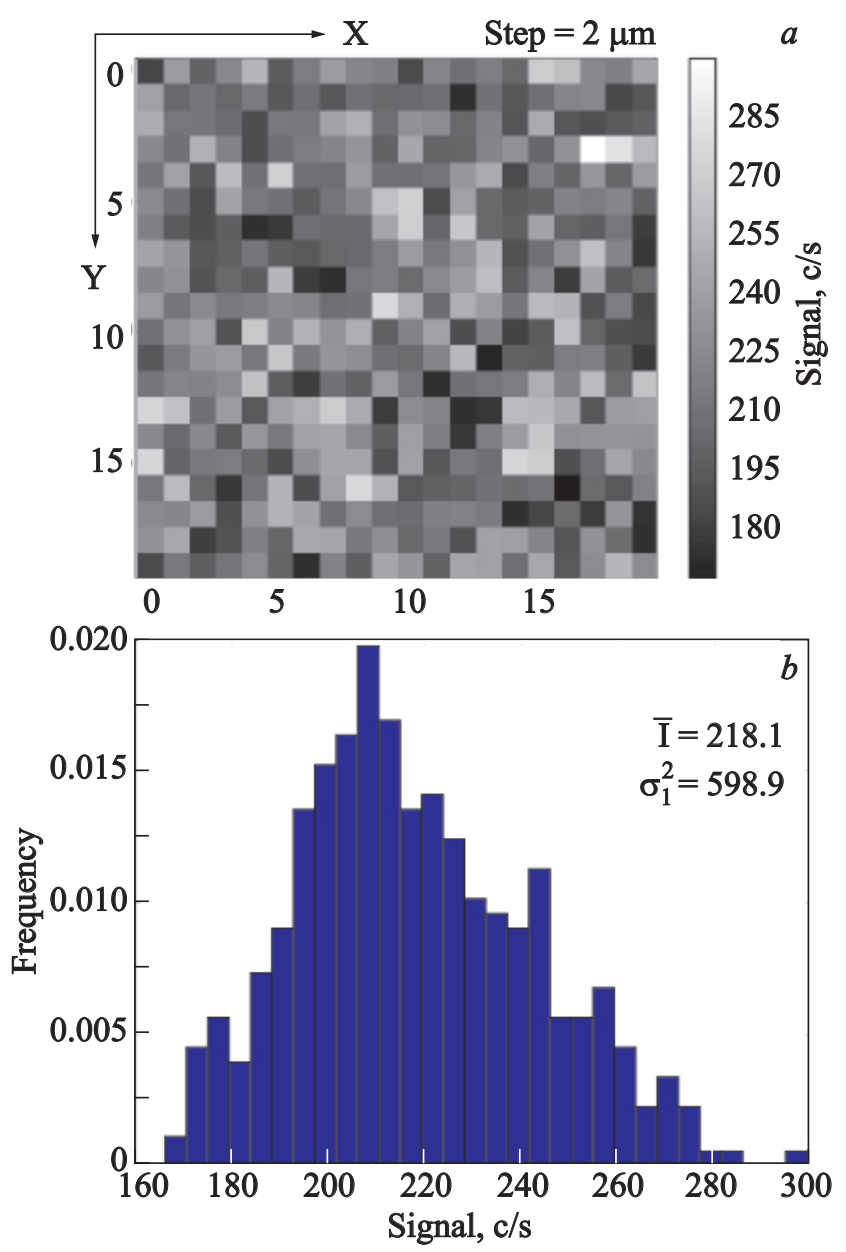

Fig. 8. (a) A map of the luminescence signal of the ZPL at $813 \mathrm{~nm}$ from the $40 \times 40 \mu \mathrm{m}(20 \times 20$ points $)$ region at $T=80 \mathrm{~K}$. (b) The histogram of the peak luminescence signal for the map (a). Statistical analyses of the map gives the average intensity $\bar{I}=218 \mathrm{c} / \mathrm{s}$ and the standard deviation $\sigma_{I}=24.3 \mathrm{c} / \mathrm{s}$. 
The spectrum of the Xe center was measured in different points of the sample surface and was used to plot a map of the luminescence intensity distribution of the primary $\mathrm{ZPL}$ at $812 \mathrm{~nm}$. The representative map of area $40 \times 40 \mu \mathrm{m}$ ( $20 \times 20$ points) is shown in Fig. $8, a$, and the corresponding histogram of the ZPL peak intensity is shown in Fig. 8,b. Statistical analyses of the experimental map gives the average intensity $\bar{I}=218 \mathrm{c} / \mathrm{s}$, the standard deviation $\sigma_{I}=$ $=24.3 \mathrm{c} / \mathrm{s}$, and the peak intensity of a single center luminescence $I_{1}=5.4 \mathrm{c} / \mathrm{s}$. In our case, the signal/noise ratio was about 100 , so all centers inside laser spot with radius $R=2 w=1.4 \mu \mathrm{m}$ contribute into the total collected signal. In correspondence with the previous analysis, the parameter $\beta=2.07$ was used to find the average number of emitting Xe centers in the laser spot: $\bar{N}=2.07\left(I / \sigma_{I}\right)^{2}=167$. At implantation dose of $10^{10}$ ion $/ \mathrm{cm}^{2}$, the number of implanted centers inside a circle with radius $R=1.4 \mu \mathrm{m}$ is $N_{\text {impl }}=616$. So, the efficiency of conversion of the implanted centers into emitting centers is estimated as $\bar{N} / N_{\text {impl }}=0.27$. Therefore, only one out of four implanted Xe centers became an optical center. This efficiency is about an order of magnitude higher than the value of 0.025 for the NV centers generation by a $14 \mathrm{keV}$ nitrogen implantation [9]. On the other hand, NV centers generation by a $2 \mathrm{MeV}$ nitrogen implantation has efficiency close to $1[8]$. We note that the experimental map at Fig. 8, $a$ was measured at condition of moderate optical saturation, so our model requires a modification that may slightly reduce the value of the estimated conversion efficiency.

The approach based on a simple Poisson distribution was used for Xe center in Ref. 26. The conversion efficiency was estimated as 1 , about four times higher than our result. This illustrates the risk of a large error in determination of this important quantity when a simplified approach is used.

In addition to the confocal micro-luminescence mapping of the uniformly implanted sample area, we performed a mapping of the transitional region between the Xe-ion implanted and the non-implanted areas. The purpose of the experiment was to study the luminescence transient profile across the implantation border and to detect and image small emitting clusters with a few Xe (or even one) emitting centers per cluster possibly located in the vicinity of the border. Conditions of the measurements were the same as described above for the uniformly implanted $\left(10^{10} \mathrm{ion} / \mathrm{cm}^{2}\right)$ area mapping, the non-implanted area was covered with the Al-foil mask while implanted.

The map is presented in Fig. 9. One can clearly see the implanted and non-implanted areas, and the transitional region. In addition, a small emitting cluster with diameter of about $2 \mu \mathrm{m}$ is visible. Comparing the cluster luminescence to the luminescence intensity of the implanted area, and accounting for the conversion efficiency of 0.27 , one may conclude that the cluster consists of about 6 emitting

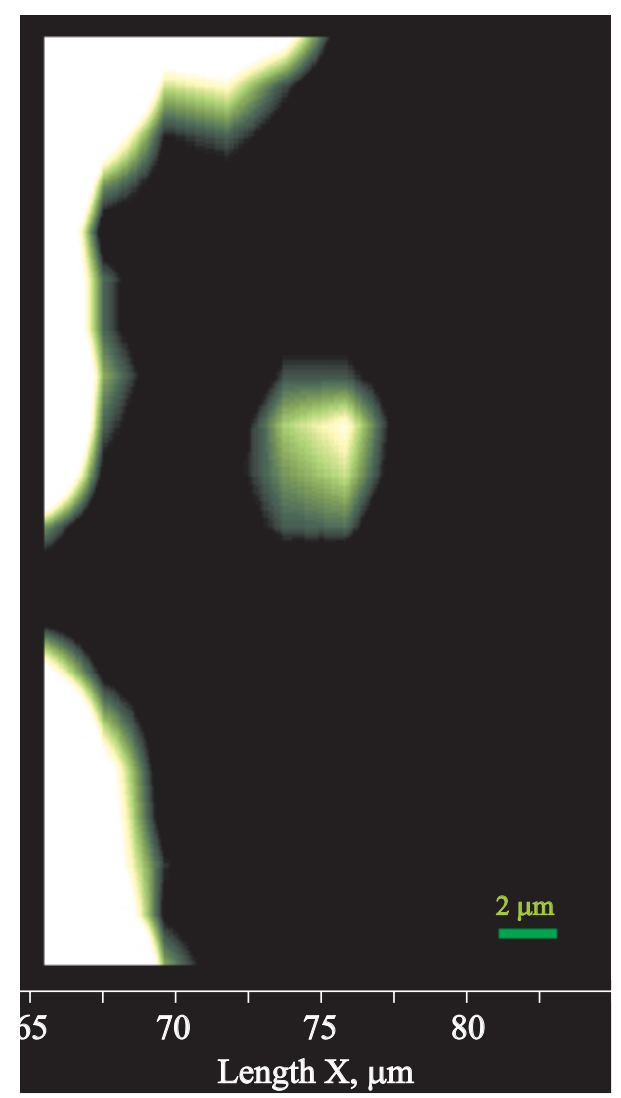

Fig. 9. A micro-luminescence map of the transitional area between the Xe-ion implanted (dose of $10^{10} \mathrm{ion} / \mathrm{cm}^{2}$ ) and nonimplanted regions based on changes in the $813 \mathrm{~nm}$ line intensity. A small cluster of emitting Xe centers is clearly visible.

and 16 non-emitting Xe centers. The origin of this cluster is unclear as it located about $10 \mu \mathrm{m}$ from the edge of the mask, which is about one order of magnitude more than predicted by SRIM simulation transversal straggling range of $1 \mu \mathrm{m}$. However, this approach may be very helpful in search for the other emitting small clusters and single centers.

\section{Conclusions}

In summary, the photoluminescence properties of the Xe-related optical center in diamond over the wide implantation dose range of $10^{10}-5 \cdot 10^{14}$ ion $/ \mathrm{cm}^{2}$ were reviewed. At low temperatures, the photoluminescence spectra features a single ZPL at $811.7 \mathrm{~nm}$ and a weak phonon sideband. The room temperature luminescence consists of a primary ZPL at $813 \mathrm{~nm}$ and a weaker line at $794 \mathrm{~nm}$. The intensity of the ZPL at $813 \mathrm{~nm}$ grows linearly with the implantation dose at doses less than $5 \cdot 10^{12} \mathrm{ion} / \mathrm{cm}^{2}$. At higher doses, the radiation-induced defects cause the nonradiative quenching of luminescence. Our experimental studies concluded that the most likely configuration of the $\mathrm{Xe}$ center is a semi-divacancy site, $\mathrm{V}-\mathrm{Xe}-\mathrm{V}$, oriented along $<111>$ direction. 
We also propose a method to determine the conversion efficiency of implanted Xe ions into emitting Xe optical centers. The technique employs the confocal microluminescence mapping and statistical analysis of the experimental results based on the compound Poisson distribution to determine number of emitting centers in the laser spot. We consider the results of numerical model simulations, and experimental micro-mapping of the region implanted with the dose of $10^{10} \mathrm{ion} / \mathrm{cm}^{2}$ based on changes in the $813 \mathrm{~nm}$ line intensity. The conversion efficiency was estimated to be 0.27 for $180 \mathrm{keV}$ implanted $\mathrm{Xe}^{+}$ions. The micro-luminescent mapping of the transitional area between the Xe-ion implanted (dose of $10^{10} \mathrm{ion} / \mathrm{cm}^{2}$ ) and non-implanted regions allowed us to image the profile of the ion distribution through the implantation boundary and to detect a small emitting cluster with a few Xe centers per cluster.

\section{Acknowledgments}

The authors thank Dr. A. Zaitsev for thermal annealing of the samples and useful discussions, Dr. Mengbing Huang for ion implantations, and Mrs. A. Bergman for participation in some measurements. The work was supported in part by PSC/CUNY.

1. J. Walker, Rep. Prog. Phys. 42, 1605 (1979).

2. A.M. Zaitsev, Optical Properties of Diamond: a Data Handbook, Springer (2001).

3. C. Kurtsiefer, S. Mayer, P. Zarda, and H. Weinfurter, Phys. Rev. Lett. 85, 290 (2000).

4. T. Gaebel, I. Popa, A. Gruber, M. Domhan, F. Jelezko, and J. Wrachtrup, New J. Phys. 6, 98 (2004).

5. C. Wang, C. Kurtsiefer, H. Weinfurter, and B. Burchard, J. Phys. B: At. Mol. Opt. Phys. 39, 37 (2006).

6. I. Aharonovich, S. Castelletto, D.A. Simpson, A. Stacey, J. McCallum, A.D. Greentree, and S. Prawer, Nano Lett. 9, 3191 (2009).

7. J. Martin, R. Wannemacher, J. Teichert, L. Bischoff, and B. Köhler, J. Appl. Phys. 75, 3096 (1999).
8. J. Meijer, B. Burchard, M. Domhan, C. Wittmann, T. Gaebel, I. Popa, F. Jelezko, and J. Wrachtrup, J. Appl. Phys. 87, 261909 (2005).

9. F. Jelezko and J. Wrachtrup, Phys. Status Solidi A203, 3207 (2006).

10. A.M. Zaitsev, A.A. Bergman, A.A. Gorokhovsky, and Mengbing Huang, Phys. Status Solidi A203, 638 (2006)

11. V.A. Martinovich, A.V. Turukhin, A.M. Zaitsev, and A.A. Gorokhovsky, J. Lumin. 102-103, 785 (2003).

12. V.A. Martinovich and A.A. Gorokhovsky, J. Lumin. 107, 261 (2004).

13. A.A. Bergman, A.M. Zaitsev, and A.A. Gorokhovsky, J. Lumin. 125, 92 (2007).

14. A.A. Bergman, A.M. Zaitsev, Mengbing Huang, and A.A. Gorokhovsky, J. Lumin. 129, 1524 (2009).

15. K. Rebane, Impurity Spectra of Solids, Plenum Press, New York (1970).

16. A.T. Collins, L. Allers, C.J.H. Wort, and G.A. Scarsbrook, Diamond and Related Materials 3, 932 (1994).

17. A.A. Gorokhovsky, A.V. Turukhin, R.R. Alfano, and W. Phillips, Appl. Phys. Lett. 66, 43 (1995).

18. W. Windl, P. Pavone, K. Karch, O. Schutt, D. Strauch, P. Giannozzi, and S. Baroni, Phys. Rev. B48, 3164 (1993).

19. P.P. Feofilov, Zh. Eksp. Teor. Fiz. 26, 609 (1954).

20. R.J. Elliott, I.G. Matthews, and E.W.J. Mitchell, Philos. Mag. 3, 360 (1958).

21. A.A. Kaplyanskii, Opt. Spectrosc. 16, 329 (1964).

22. A.B. Anderson and E.J. Grantscharova, Phys. Rev. B54, 14341 (1996).

23. A.D. Greentree, P. Olivero, M. Draganski, E. Trajkov, J.R. Rabeau, P. Reichart, B.C. Gibson, S. Rubanov, S.T. Huntington, D.N. Jamieson, and S. Prawer, J. Phys.: Condens. Matter 18, S825 (2006).

24. SRIM-2003: http://www.srim.org.

25. E.H. Lloyd, Probability, Handbook of Applicable Mathematics, John Wiley\&Sons (1980), ch. 14.

26. Chunlang Wang, A Solid-State Single Photon Source Based on Color Centers in Diamond, Dissertation, Munich (2007). 